\title{
Changes in macular drusen parameters preceding the development of neovascular age-related macular degeneration
}

\author{
Ali Lamin ${ }^{1,2} \cdot$ Adam M. Dubis ${ }^{1,2} \cdot$ Sobha Sivaprasad $\mathbb{C}^{1,2}$ \\ Received: 11 July 2018 / Revised: 10 December 2018 / Accepted: 22 December 2018 / Published online: 24 January 2019 \\ (c) The Royal College of Ophthalmologists 2019
}

\begin{abstract}
Purpose To assess the rate of change in macular drusen load in fellow eyes of patients with unilateral neovascular agerelated macular degeneration (AMD) to evaluate whether the change in drusen load determines the onset and type of choroidal neovascularisation $(\mathrm{CNV})$.

Methods Subjects with unilateral neovascular AMD with a minimum of 2 years follow-up were identified retrospectively from the hospital electronic database. Drusen count, area and volume measurements at the macula of the contralateral eye were recorded using the commercial software on the Topcon 3D OCT-2000 devices over the previous 2 years. The mean rate of change of these parameters over time was compared between fellow eyes that converted to various CNV subtypes and those that did not.

Results Two hundred forty-eight patients met the inclusion criteria. Of these patients, 179 patients did not progress to neovascular AMD (Group 1) while 69 patients converted (Group 2) at the end of 2 years follow up. Mean drusen volumes and drusen areas increased significantly in Group 2 in the 2 nd year by $0.031 \mathrm{~mm}^{3}(p<0.001)$ and $0.572 \mathrm{~mm}^{2}(p=0.002)$, respectively. However, there was no difference in the rate of change between the two groups at year 1 . Furthermore, for each 0.1 $\mathrm{mm}$ increase in the cubed root of baseline mean drusen volume increases the odds of progressing to CNV by $40 \%$ (95\% CI 1.2 $1.6 ; p<0.001)$. The increase in drusen volume was higher in the occult CNV group compared to classic CNV $(p=0.048)$. Conclusion A significant increase in mean drusen volume occurs in eyes in the preceding 12 months prior to conversion to neovascular AMD and this change is more significant in eyes with occult CNV.
\end{abstract}

\section{Introduction}

Drusen are extracellular focal deposits that accumulate between the basal lamina of the retinal pigment epithelium (RPE) and the inner collagenous layer of the Bruch's membrane. They appear as yellowish white deposits on biomicroscopy and colour fundus photographs and have a predilection for the macula $[1,2]$.

Drusen vary in size. They are the hallmark of age-related macular degeneration (AMD). The classification of AMD is based on the size of drusen [3]. Small drusen or druplets defined as $\leq 63 \mu \mathrm{m}$ in size have a low risk of progression to

Sobha Sivaprasad

sobha.sivaprasad@moorfields.nhs.uk

1 NIHR Moorfields Biomedical Research Centre, Moorfields Eye Hospital NHS Foundation Trust, London, UK

2 UCL Institute of Ophthalmology, London, UK advanced AMD. Early AMD is characterised by the presence of medium-sized drusen ( $>63 \mu \mathrm{m}$ to $\leq 125 \mu \mathrm{m}$ ). The presence of large drusen defined as size of $>125 \mu \mathrm{m}$ or more categorises the eye to having intermediate AMD [3, 4]. These eyes with intermediate AMD are at risk of progression to advanced AMD developing either choroidal neovascularisation $(\mathrm{CNV})$ or geographic atrophy $(\mathrm{GA})$ or both $[5,6]$. The risk of development of $\mathrm{CNV}$ in the second eye in patients with unilateral advanced AMD is variable and can range from 20 to $50 \%$ over 5-10 years [7-9]. When considering the $\mathrm{CNV}$ subtypes, occult $\mathrm{CNV}$ are more likely to be associated with intermediate drusen than pure classic CNV that may occur in eyes with no or small drusen [10]. We evaluated the mean change in drusen load in eyes that convert to neovascular AMD to study whether the change in drusen volume may be a risk factor for conversion to neovascular AMD, especially in eyes with occult CNV.

Although size and area of large drusen are established risk factors, the course of each druse within the macula vary and therefore a global measure of drusen load may be a 
more appropriate risk factor of AMD. Optical coherence tomography (OCT) provides in vivo cross-sectional images of different retinal structures, that is, analogues to the histological structure. Recently developed spectral-domain OCT (SD-OCT) technology provides real-time, highresolution images of the macular area in two and three dimensions $[11,12]$. Using automated segmentation algorithms, SD-OCT is also able to delineate drusen and longitudinally track changes in macular total drusen count, global area and volume occupied by drusen in AMD eyes [13-15].

The SD-OCT algorithm defines drusen as a degree of deformity in the RPE [13, 16, 17]. Consequently, small drusen are not detected by OCT as they do not elevate RPE layer considerably. As a result, drusen count and area are underestimated by SD-OCT compared to fundus photography $[16,18,19]$. However, the volume of drusen is measured reliably by SD-OCT, which render this technique superior to colour fundus photography [15].

The ability of SD-OCT to measure drusen count, area and volume encouraged some groups of researchers to study the effect of these parameters on disease progression to advanced AMD (CNV or GA). Several reports have been published in this regard with variable results [20-24]. Most of these studies have been evaluated using the Cirrus OCT (Zeiss, USA) algorithm. The Topcon SD-OCT also has an in-built automated drusen analysis algorithm but has very limited publications on its use. Moreover, the detection limit of drusen is different with Cirrus OCT compared to Topcon OCT. As Topcon OCT is widely used in daily practice, it is important to evaluate whether the Topcon drusen analysis algorithm provides the same outcomes as other similar algorithms over time. In this study, we quantified the drusen load in terms of numbers, area and volume using the automated Topcon drusen analysis algorithm in fellow eyes of patients with unilateral neovascular AMD.

Our objectives were to evaluate whether the change in drusen count, area or volume as measured using the automated drusen analysis algorithm can predict conversion to $\mathrm{CNV}$ and if so, whether any of these parameters predicted the type of CNV.

\section{Methods}

\section{Data collection}

This retrospective study was approved by Moorfields Review Board (ROAD 17/004) for evaluation of data and images obtained in routine clinical practice. The research followed the tenets of the Declaration of Helsinki. Informed consent was not required for this study. Patients with unilateral neovascular AMD and early or intermediate AMD in the fellow eye who were imaged using SD-OCT in both eyes over 2 years were identified from the hospital database. Those patients were divided into two groups: nonprogressors who did not convert from early/intermediate to advanced AMD $(n=179)$; and those who progressed to wet AMD $(n=69)$. In Group 1, the OCT scans taken at baseline, year 1 and around the 2-year follow-up were used for the analysis. In Group 2, the OCTs used in the analysis included those performed up to 2 years prior to the last clinic visit before the development of CNV. Eyes with GA were excluded from the study as we know from a previous study that drusen volume was a predicator of conversion to CNV and not GA [25]. Also, eyes with, vitelliform lesions, other macular pathology or poor quality OCT scan images were excluded from the study.

\section{OCT scans}

All eyes had three SD-OCT scan images (baseline, year 1 and year 2) using 3D OCT-2000 (Topcon, Tokyo, Japan). The 3D OCT-2000 had a $6 \mu \mathrm{m}$ axial image resolution and imaging speed of 18,000 axial scans per second. On the 3D OCT-2000, the 3D macular scan used was 128 line raster with 512 A-scans each, within $6 \times 6 \mathrm{~mm}^{2}$. Automated quantification of drusen count, area and volume within the 6-mm cube centred on the fovea were obtained using the Topcon software version 2.00. The algorithm on 3D OCT2000 , which was validated in a previous study [14], defines drusen based on calculating the difference between the elevated RPE caused by drusen and a virtual line representing the presumed Bruch's membrane. This study also showed that small drusen were poorly detected. Topcon 3D OCT-2000 has a smallest drusen detection limit of $340 \mu \mathrm{m}$ [26].

\section{Fundus fluorescein angiography (FFA)}

Available FFA images of patients that converted to neovascular AMD were graded for CNV subtypes. As pure or predominantly classic and occult $\mathrm{CNV}$ were the most common CNV subtypes compared to retinal angiomatous proliferation (RAP), the drusen volume change at the visit prior to the conversion was compared between eyes that converted to classic versus occult CNVs.

\section{Statistical analysis}

Baseline characteristics were analysed using descriptive statistics. Normality was tested by the Shapiro-Wilk Test. As the data was not normally distributed and to adjust for baseline drusen parameters, we transformed the drusen area and volume to square root and cubic root as previously reported [15, 20-22]. We also assessed the variables that 
Table 1 Comparing baseline drusen count, area and volume between eyes that developed $\mathrm{CNV}$ and eyes that did not develop

\begin{tabular}{llll}
\hline Drusen measurements & $\begin{array}{l}\text { Non-CNV-developed eyes } \\
(n=179)\end{array}$ & $\begin{array}{l}\text { CNV-developed eyes } \\
(n=69)\end{array}$ & $p$-Value \\
\hline $\begin{array}{l}\text { Mean drusen count }(\mathrm{SD}) \\
\begin{array}{l}\text { Mean drusen square root area } \\
(\mathrm{SD})\end{array}\end{array}$ & $5.42(6.67)$ & $9.57(7.41)$ & $<0.001$ \\
$\begin{array}{l}\text { Mean drusen cube root volume } \\
(\mathrm{SD})\end{array}$ & $0.8101 \mathrm{~mm}(0.79)$ & $1.2162 \mathrm{~mm}(0.58)$ & $<0.001$ \\
\hline
\end{tabular}

influenced the change in drusen parameters over the 2 years and the gradient of change over 1 and 2 years. The baseline measurements were also analysed as predicators of progression to $\mathrm{CNV}$ using logistic regression analysis. Change in drusen count, area and volume measurements from baseline to year 1 (first year) and from year 1 to year 2 (second year) were compared between the two groups with the Mann-Whitney $U$ test. We also compared drusen parameter change in the eyes that converted to occult or classic CNV using independent-samples $t$-test. Statistical analysis was performed using the SPSS software (version 24) and a $p$-value of $\leq 0.05$ was considered statistically significant.

\section{Results}

\section{Patients}

After reviewing the medical records of 1671 patients who were treated with anti-VEGF therapy at Moorfields Eye Hospital between August 2008 and September 2016, 248 patients who met the inclusion criteria were identified. In particular, we required 2 years of follow-up data to study the change in drusen parameters at 12 and 24 months preceding the onset of CNV in the fellow eye. We excluded all patients who did not have an OCT scan done at $12 \pm$ 2 months and $24 \pm 2$ months follow-up. We also excluded patients with at least one of the following: SD-OCT scans with an instrument image quality metric $<30$; scans with poor foveal centration and presence of artefacts. Among the 248 patients involved in the study, $161(65 \%)$ were females and $87(35 \%)$ were males with a mean age of 73.5 years (SD, 8.6). One hundred ninety-nine had intermediate AMD and 49 had early AMD. These eyes were divided into two groups: non-CNV-developed eyes (72\%, $n=179)$ and CNV-developed eyes $(28 \%, n=69)$.

\section{Baseline drusen count, area and volume measurements}

At baseline, the mean (SD) drusen count, drusen area and drusen volume were $6.58(7.1), 1.42 \mathrm{~mm}^{2}$ (1.85) and
Table 2 Assessing predictors for $\mathrm{CNV}$ progression using logistic regression analysis

\begin{tabular}{llll}
\hline & Odds ratio $^{\mathrm{a}}$ & $95 \%$ CI & $p$-Value \\
\hline Age & 1.067 & $1-1.1$ & $<0.001$ \\
Sex & 2.02 & $1-3.7$ & 0.027 \\
Drusen count & 1.078 & $1-1.1$ & $<0.001$ \\
$\begin{array}{l}\text { Square root drusen area, } \mathbf{0 . 1} \mathbf{~ m m} \\
\text { increase }\end{array}$ & 1.069 & $1-1.1$ & $<0.001$ \\
$\begin{array}{l}\text { Cube root drusen volume, } \mathbf{0 . 1} \mathbf{~ m m} \\
\text { increase }\end{array}$ & 1.4 & $1.2-1.6$ & $<0.001$ \\
\hline
\end{tabular}

${ }^{\mathrm{a}}$ Odds ratios not adjusted

$0.06 \mathrm{~mm}^{3}(0.09)$, respectively. The square root drusen area and cube root drusen volume were $0.9231 \mathrm{~mm}(0.76)$ and $0.288 \mathrm{~mm}(0.2)$, respectively. Baseline drusen count, square root area and cube root volume measurements were compared between the two groups (69 CNV-developed eyes versus 179 non-CNV-developed eyes) using the MannWhitney $U$ test. These three baseline drusen parameters (count, area and volume) were greater in CNV-developed eyes than non-CNV-developed as shown in Table 1.

\section{Predication of progression to CNV based on baseline drusen measurements}

Baseline count, area and volume measurements as well as age and sex were assessed as predictors of progression to CNV (Table 2). Logistic regression analysis of these drusen measurements revealed that baseline volume was a significant predictor for developing $\mathrm{CNV}$ at 2 years of follow-up. Each $0.1 \mathrm{~mm}$ increase in the cubed root of baseline drusen volume increases the odds of progressing to CNV by $40 \%$ (95\% CI 1.2-1.6; $p<0.001)$.

\section{Change in drusen measurements over two time points (year 1 and year 2)}

We also compared the change rate of drusen count, area and volume measurements between the two groups (progressed to $\mathrm{CNV}$ (Group 2) and non-progressed (Group 1)) during the 1st year and 2nd year using Mann-Whitney $U$ test (Table 3). The change rate between the two groups in the 
Table 3 Comparing the change in drusen count, area and volume between the two groups in the first and second year

\begin{tabular}{lllll}
\hline & & Group 1 $(n=179)$ & Group 2 $(n=69)$ & $p$-Value \\
\hline Year 1 & Count change & 1.15 & 1.33 & 0.484 \\
& Square root area change & $0.12(0.27)$ & $0.11(0.24)$ & 0.902 \\
& Cube root volume change & $0.02(0.08)$ & $0.02(0.07)$ & 0.834 \\
Year 2 & Count change & 0.69 & 1.64 & 0.052 \\
& Square root area change & $0.12(0.27)$ & $0.19(0.27)$ & 0.027 \\
& Cube root volume change & $0.03(0.09)$ & $0.05(0.07)$ & 0.019 \\
\hline
\end{tabular}

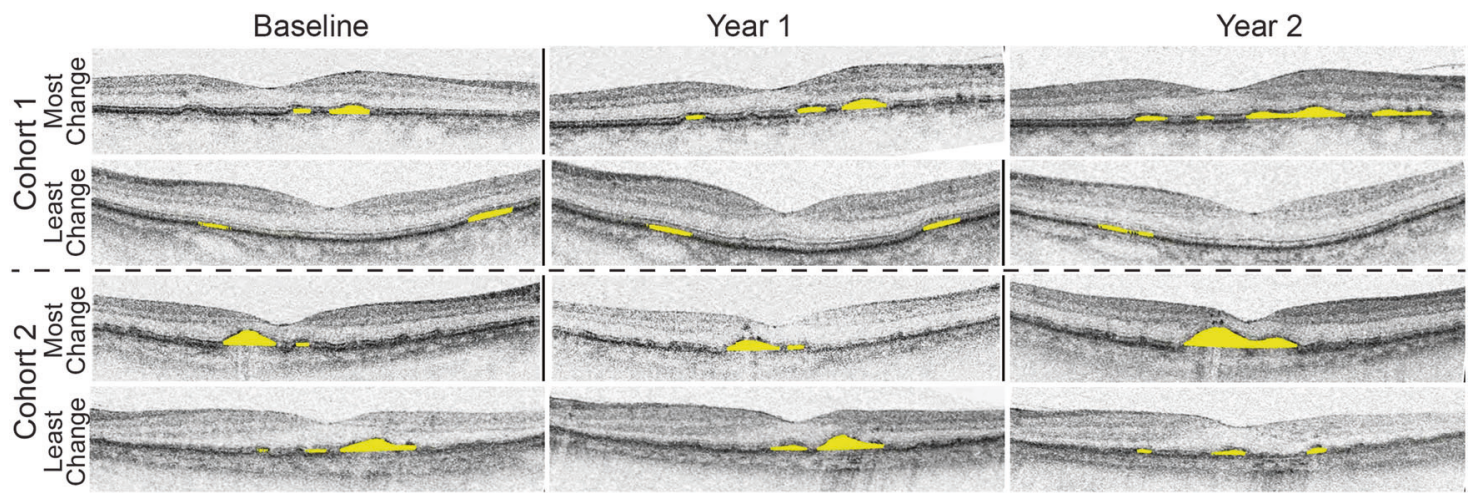

Fig. 1 Change in drusen volume from baseline to 2 years. Shown here are the foveal scans for the subjects with the greatest and least change from baseline to year 2 for each cohort. The yellow is what the Topcon

2nd year was statistically significant, particularly drusen volume $(p=0.019)$ and area $(p=0.027)$. However, the rate of change between the two groups in the 1st year was not statistically significant. Change in drusen morphology is shown in Fig. 1.

Figure 2 shows the gradient at which the overall drusen volume changed in the two groups and the data shows that the total drusen volume increases steeply in the year preceding the development of CNV.

We also compared the change in these drusen parameters in eyes that converted to neovascular AMD. Fifty patients had FFA and the CNV subtypes include 16 (32\%) classic and predominantly classic CNV, 32 (64\%) occult CNV and 2 (4\%) RAP. As RAP lesions were rare, we compared the differences in drusen parameters between the classic and occult subtypes. While drusen parameters were alike in both $\mathrm{CNV}$ groups, the rate of change of drusen volume was higher in the occult group in the year preceding the development of CNV $(p=0.048)$.

\section{Discussion}

This study confirms that an acceleration of total drusen volume on SD-OCT occurs prior to the conversion to neovascular AMD in the fellow eyes of patients with unilateral neovascular AMD. This accelerated increase in total drusen volume occurs in the year preceding the onset of
OCT designated as drusen volume for the scan. Not that least change could also be a decrease in volume (top set)

$\mathrm{CNV}$. The mean rate of increase in cube root drusen volume is $0.05 \mathrm{~mm}$ in the year preceding the development of $\mathrm{CNV}$, compared with the first year follow-up of the same group as well as the non-CNV-developed group that had a mean growth rate of cubic root drusen volume to be 0.02-0.03 $\mathrm{mm}$ per year. The cube root transformation of drusen volume and the square root transformation of drusen area were performed to eliminate the dependence of the drusen growth rate on the baseline drusen size. This can be applied to clinical practice because we can counsel patients more accurately about the time to second eye involvement.

The study also shows that the patients with increased drusen growth rate may form an enriched cohort for clinical trials evaluating preventive measures for the development of $\mathrm{CNV}$ as the event rate for this cohort will be higher than recruiting any patient with unilateral $\mathrm{CNV}$ when the reported probability of occurrence of $\mathrm{CNV}$ in the fellow eye is only around $10-12 \%$ per year. It will also shorten the duration of the clinical trial. A previous study on drusen volume showed that recruiting patients with a baseline volume of $>0.03 \mathrm{~mm}^{3}$ or $0.31 \mathrm{~mm}$ cube root volume in the central $3 \mathrm{~mm}$ diameter would result in $50 \%$ showing an increase in baseline volume and would enrich a prevention trial cohort and shorten the trial duration [23]. Adding a parameter of drusen growth rate of $0.05 \mathrm{~mm}$ cube root volume across $6 \mathrm{~mm}$ diameter of the macula centred at the fovea will further reduce the sample size. 

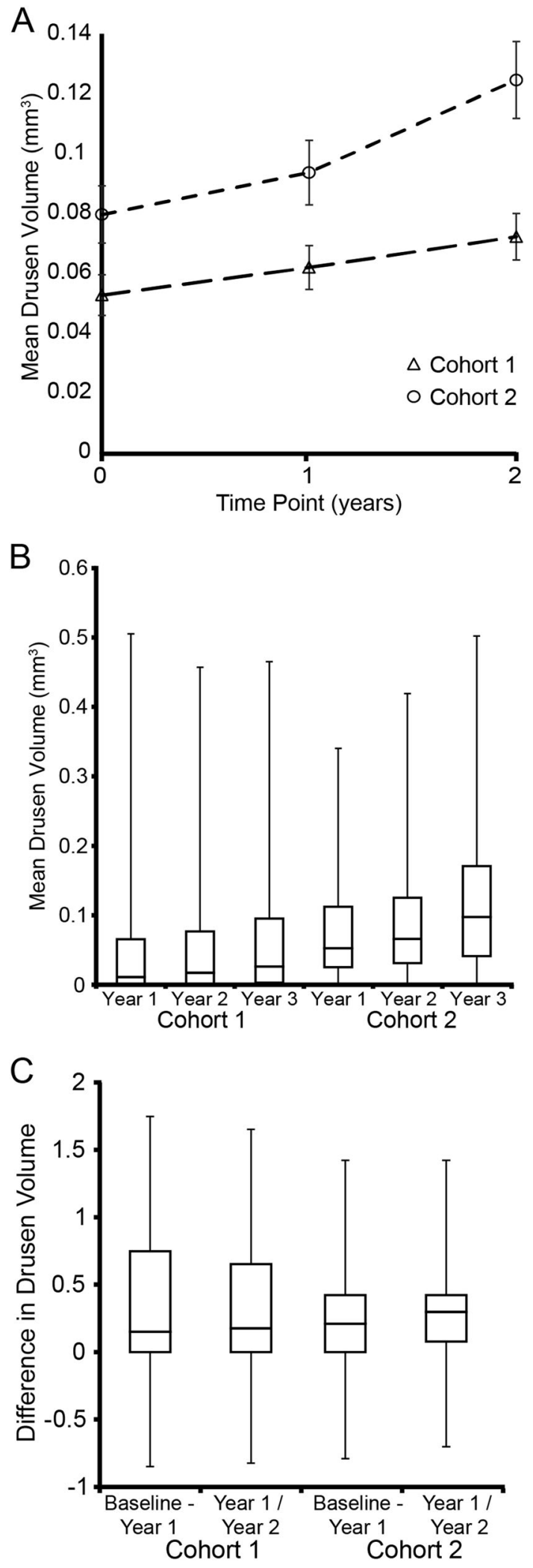

In addition, this study shows that the rate of change of drusen volume was higher in eyes that developed occult $\mathrm{CNV}$ in the year preceding the development of CNV. There
Fig. 2 Mean volume and change from baseline to year 2 in those with and without CNV. a Shows the mean drusen volume, error bars or SEM. b Shows the distributions of drusen volumes. While on average that with CNV had more drusen volume this was not absolute with the ranges overlapping nearly entirely. c On average those who went on to develop CNV had a greater increase in drusen volume, however again there was considerable overlap with those who did not

was no difference in other drusen parameters in terms of drusen count, area and volume. Previous studies reported that the clinical features in the fellow eye correlate with the type of $\mathrm{CNV}$ in the affected eye in AMD patients with unilateral CNV [10, 24]. Abugreen et al. [10] found that the fellow eyes of occult CNV have significantly more severe AMD features compared to the eyes with classic CNV. Sivaprasad et al. [24] showed that there is an association between the proportion of occult CNV in the affected eye and the severity of the disease in the fellow eye of Caucasians compared to the Chinese patients.

To our knowledge, our study is the first in being used 3D OCT-2000 algorithm to study drusen quantification longitudinally and the largest study (sample size) of drusen volume for AMD patients who developed CNV $(n=69)$. This could be one of the strengths of our study as the Topcon commercial software is available for everyone to use, rather than a specific research centre developed software.

Limitations of this study are the use of commercially available software that does not allow user correction of segmentation. While theoretically a limitation, this limitation is surpassed by the essential nature of the work. It is essential understanding for clinical care as SD-OCT segmentation and drusen identification correction are not currently cost/time-effective in clinical practice. Therefore, understanding of change based on 'raw' outputs is needed. Additionally, the inherent differences between devices make comparisons between devices not possible and therefore requires each device to be independently verified. These differences result in two categories of differences. First, we did not subdivide drusen measurements into smaller macular subfield within the total macular field of 6 $\mathrm{mm}$ cube as the algorithm did not permit this analysis. Updated versions of this software are anticipated to provide this analysis. However, the study shows that the results obtained on drusen volume in $6 \mathrm{~mm}$ cube parallels that obtained in $3 \mathrm{~mm}$ central macula and can be used as a predictor of CNV. We also did not adjust for other known risk factors of disease progression such as smoking, raised BMI and genetic factors. We also excluded a large proportion of patients for whom scans were not available at the strict time-points planned for this study. The rationale for establishing a standardised 2-year follow-up interval was based on 2-year study endpoints, which showed that a 
greater baseline drusen volume was predicative of an increased 2-year progression to CNV [25].

In summary, this study has shown that drusen volume growth rate across a $6 \mathrm{~mm}$ macula cube using the automatic Topcon drusen analysis software may be used as a predictor for conversion to CNV especially occult type. Serial OCTs over a shorter time interval may provide better information than the two time points that we have chosen.

\section{Summary}

\section{What was known before}

- Baseline drusen volume appears to be a significant risk factor for progression to late AMD.

\section{What this study adds}

- Drusen volume and its growth rate are higher in the preceding 12 months prior to conversion to neovascular AMD, particularly occult type, than those did not develop CNV. These findings may be used as a predictor for conversion to $\mathrm{CNV}$ using SD-OCT.

\begin{abstract}
Acknowledgements The research was supported by the National Institute for Health Research (NIHR) Biomedical Research Centre based at Moorfields Eye Hospital NHS Foundation Trust and UCL Institute of Ophthalmology. The views expressed are those of the author(s) and not necessarily those of the NHS, the NIHR or the Department of Health.
\end{abstract}

\section{Compliance with ethical standards}

Conflict of interest The authors declare that they have no conflict of interest.

Publisher's note: Springer Nature remains neutral with regard to jurisdictional claims in published maps and institutional affiliations.

\section{References}

1. Sarks JP, Sarks SH, Killingsworth MC. Evolution of soft drusen in age-related macular degeneration. Eye. 1994;8:269-83.

2. van der Schaft TL, Mooy CM, de Bruijn WC, Oron FG, Mulder PG, de Jong PT. Histologic features of the early stages of agerelated macular degeneration. A statistical analysis. Ophthalmology. 1992;99:278-86.

3. Ferris FL 3rd, Wilkinson CP, Bird A, Chakravarthy U, Chew E, Csaky K, et al. Clinical classification of age-related macular degeneration. Ophthalmology. 2013;120:844-51.

4. Ferris FL, Davis MD, Clemons TE, Lee LY, Chew EY, Lindblad AS, et al. A simplified severity scale for age-related macular degeneration: AREDS Report No. 18. Arch Ophthalmol. 2005;123:1570-4.

5. Klein R, Klein BE, Jensen SC, Meuer SM. The five-year incidence and progression of age-related maculopathy: the Beaver Dam Eye Study. Ophthalmology. 1997;104:7-21.

6. Klein R, Klein BE, Tomany SC, Meuer SM, Huang GH. Ten-year incidence and progression of age-related maculopathy: the Beaver Dam eye study. Ophthalmology. 2002;109:1767-79.

7. Macular Photocoagulation Study Group. Five-year follow-up of fellow eyes of patients with age-related macular degeneration and unilateral extrafoveal choroidal neovascularization. Arch Ophthalmol. 1993;111:1189-99.

8. Wang JJ, Rochtchina E, Lee AJ, Chia EM, Smith W, Cumming $\mathrm{RG}$, et al. Ten-year incidence and progression of age-related maculopathy: the blue Mountains Eye Study. Ophthalmology. 2007;114:92-8.

9. Macular Photocoagulation Study Group. Risk factors for choroidal neovascularization in the second eye of patients with juxtafoveal or subfoveal choroidal neovascularization secondary to age-related macular degeneration. Arch Ophthalmol. 1997; 115:741-7.

10. Abugreen S, Muldrew KA, Stevenson MR, VanLeeuwen R, DeJong PT, Chakravarthy U. CNV subtype in first eyes predicts severity of ARM in fellow eyes. Br J Ophthalmol. 2003; 87:307-11.

11. Srinivasan VJ, Wojtkowski M, Witkin AJ, Duker JS, Ko TH, Carvalho M, et al. High-definition and 3-dimensional imaging of macular pathologies with high-speed ultrahigh-resolution optical coherence tomography. Ophthalmology. 2006;113:2054.

12. Wojtkowski M, Srinivasan V, Fujimoto JG, Ko T, Schuman JS, Kowalczyk A, et al. Three-dimensional retinal imaging with highspeed ultrahigh-resolution optical coherence tomography. Ophthalmology. 2005;112:1734-46.

13. Schlanitz FG, Ahlers C, Sacu S, Schutze C, Rodriguez M, Schriefl $\mathrm{S}$, et al. Performance of drusen detection by spectral-domain optical coherence tomography. Invest Ophthalmol Vis Sci. 2010;51:6715-21.

14. Iwama D, Hangai M, Ooto S, Sakamoto A, Nakanishi H, Fujimura $\mathrm{T}$, et al. Automated assessment of drusen using three-dimensional spectral-domain optical coherence tomography. Invest Ophthalmol Vis Sci. 2012;53:1576-83.

15. Gregori G, Wang F, Rosenfeld PJ, Yehoshua Z, Gregori NZ, Lujan BJ, et al. Spectral domain optical coherence tomography imaging of drusen in nonexudative age-related macular degeneration. Ophthalmology. 2011;118:1373-9.

16. Diniz B, Ribeiro R, Heussen FM, Maia M, Sadda S. Drusen measurements comparison by fundus photograph manual delineation versus optical coherence tomography retinal pigment epithelial segmentation automated analysis. Retina. 2014;34:55-62.

17. Jain N, Farsiu S, Khanifar AA, Bearelly S, Smith RT, Izatt JA, et al. Quantitative comparison of drusen segmented on SD-OCT versus drusen delineated on color fundus photographs. Invest Ophthalmol Vis Sci. 2010;51:4875-83.

18. Gregori G, Yehoshua Z, Filho CAAG, Sadda SR, Nunes RP, Feuer WJ, et al. Change in drusen area over time compared using spectral-domain optical coherence tomography and color fundus imaging. Invest Ophthalmol Vis Sci. 2014;55:7662-8.

19. Yehoshua Z, Gregori G, Sadda SR, Penha FM, Goldhardt R, Nittala MG, et al. Comparison of drusen area detected by spectral domain optical coherence tomography and color fundus imaging. Invest Ophthalmol Vis Sci. 2013;54:2429-34.

20. Yehoshua Z, Wang F, Rosenfeld PJ, Penha FM, Feuer WJ, Gregori G., et al. Natural history of drusen morphology in age-related macular degeneration using spectral domain optical coherence tomography. Ophthalmology. 2011;118:2434-41. 
21. Abdelfattah NS, Zhang H, Boyer DS, Rosenfeld PJ, Feuer WJ, Gregori G, et al. Drusen volume as a predictor of disease progression in patients with late age-related macular degeneration in the fellow eye. Invest Ophthalmol Vis Sci. 2016;57:1839-46.

22. Garcia Filho CA, Yehoshua Z, Gregori G, Nunes RP, Penha FM, Moshfeghi AA, et al. Change in drusen volume as a novel clinical trial endpoint for the study of complement inhibition in agerelated macular degeneration. Ophthalmic Surg Lasers Imaging. 2014;45:18-31.

23. Schaal KB, Rosenfeld PJ, Gregori G, Yehoshua Z, Feuer WJ. Anatomic clinical trial endpoints for nonexudative age-related macular degeneration. Ophthalmology. 2016;123:1060-79.
24. Sivaprasad S, Membrey WL, Sivagnanavel V, Gonzalez JG, Liu DT, Chan WM, et al. Second eye of patients with unilateral neovascular age-related macular degeneration: Caucasians vs Chinese. Eye. 2006;20:923-6.

25. Folgar FA, Yuan EL, Sevilla MB, Chiu SJ, Farsiu S, Chew EY, et al. Drusen volume and retinal pigment epithelium abnormal thinning volume predict 2-year progression of age-related macular degeneration. Ophthalmology. 2016;123:39-50.

26. Schlanitz FG, Baumann B, Spalek T, Schutze C, Ahlers C, Pircher $\mathrm{M}$, et al. Performance of automated drusen detection by polarization-sensitive optical coherence tomography. Invest Ophthalmol Vis Sci. 2011;52:4571-9. 